\title{
Value of Dyna CT in guiding embolization during transarterial uterine artery embolization of fibroids
}

\author{
CAO-YE WANG $^{1 *}$, JIN-GUO XIA $^{2 *}$, WEN-HUA CHEN $^{1}$, YI-FENG LU ${ }^{1}$, ZONG-HONG HAN $^{1}$ and QI WANG \\ ${ }^{1}$ Department of Interventional Radiology, The First People's Hospital of Changzhou, Changzhou, Jiangsu 213003; \\ ${ }^{2}$ Hepatobiliary Center, The First Affiliated Hospital of Nanjing Medical University, Nanjing, Jiangsu 210009, P.R. China
}

Received April 25, 2019; Accepted March 17, 2020

DOI: $10.3892 /$ etm.2020.8875

\begin{abstract}
The present study aimed to assess the usefulness of Dyna CT during transarterial uterine artery embolization (UAE) of fibroids. A total of 65 patients with symptomatic submucosal and intramural fibroids scheduled for transarterial UAE at the First People's Hospital of Changhou between May 2016 and September 2018 were included. Dyna CT and routine digital subtraction angiography (DSA) were performed in all patients during angiographic embolization of the bilateral internal iliac arteries. The visualization qualities of uterine artery origin and fibroids, as imaged by Dyna CT, were compared with DSA anterior-posterior images. Dyna CT provided excellent 3-dimensional visualization of vascular structures of the bilateral internal iliac arteries and uterine artery origin. Dyna CT was primarily useful in patients with overlapped uterine arteries or complex vascular anatomies on DSA anterior-posterior images (Dyna CT 100\% vs. DSA anterior-posterior $69.2 \%, \mathrm{P}=0.03)$. However, stained fibroids were identified in $57 / 65$ cases $(87.7 \%)$ by Dyna CT, and in all of the cases $(100 \%)$ by DSA anterior-posterior imaging $(\mathrm{P}=0.03)$. Dyna CT is not suitable for procedural evaluation when used alone for transarterial UAE of fibroids. However, Dyna CT is an excellent supplement to DSA anterior-posterior imaging, as it provides additional information to aid in treatment planning.
\end{abstract}

Correspondence to: Dr Qi Wang, Department of Interventional Radiology, The First People's Hospital of Changzhou, 185 Juqian Road, Changzhou, Jiangsu 213003, P.R. China

E-mail: 15861174247@139.com

${ }^{*}$ Contributed equally

Abbreviations: UAE, transarterial uterine artery embolization; DSA, routine digital subtraction angiography; 3-D, 3-dimensional; DSA-AP, digital subtraction angiography anterior-posterior

Key words: Dyna CT, dynamic CT angiography, vascular anatomy, transarterial uterine artery embolization, fibroids

\section{Introduction}

Uterine fibroids, commonly referred to as myomas or leiomyomas, are the most common benign lesion occurring in females of child-bearing age. While the majority of uterine fibroids are benign, they may negatively affect the female reproductive system, reducing fertility and causing significant morbidity for symptomatic patients, which may decrease their overall quality of life $(1,2)$. It is estimated that $25 \%$ of females of reproductive age develop uterine fibroids (3). At present, there are limited non-surgical options for the long-term treatment of uterine fibroids. While surgery is the primary and most effective treatment, uterine fibroids have high recurrence rates after treatment, leading to psychological and economic burdens to the patients and society at large $(4,5)$. Uterine artery embolization (UAE) is a safe and highly effective minimally invasive approach for treating symptomatic patients (6). However, this approach exposes the uterus and ovaries to high levels of ionizing radiation, which may negatively affect the child-bearing ability of females. In addition, UAE is a complex procedure requiring skilled and well-trained interventionists (7). In recent years, several techniques have been proposed for reducing the dosage of ionizing radiation, including bilateral femoral access and low imaging frame rates (8). However, these techniques may lead to low image quality and an increased risk of injury to the patient.

During the UAE procedure, imaging guidance is of critical importance to increase the accuracy of UAE and minimize any potential complications associated with this highly complex procedure $(9,10)$. Image guidance under 2-dimentional (2-D) digital subtraction angiography (DSA) for UAE is widely reported in the literature $(7,11)$.

However, with the development of new imaging modalities, it has become possible to obtain optimal images using enhanced 3-D functional and axial-anatomic adjunctive technologies $(12,13)$. Dyna CT, which is also known as modern C-arm $\mathrm{CT}$ or cone-beam CT angiography, uses all of the capabilities of modern C-arm digital angiography systems commonly available in endovascular suites. Dyna CT has become increasingly adopted in the clinical community due to its high image quality, versatility and dedicated applications for planning, guiding, monitoring and assessing interventional procedures (14). In a previous study, the usefulness of 3-D angiography was demonstrated using Dyna CT in the treatment of hepatocellular 
carcinoma via transcatheter arterial chemoembolization (15). In addition, 3-D angiography using Dyna CT was also reported to be useful in diagnostic and interventional radiology procedures involving the head, specifically cerebral aneurysms (16).

Despite the apparent advantages of Dyna CT, the use of imaging technology for UAE of fibroids has rarely been reported. It was hypothesized that Dyna CT may be a valuable imaging tool for improving the treatment of fibroids during UAE. The present study aimed to determine whether 3-D angiography by Dyna CT may replace conventional digital subtraction angiography anterior-posterior imaging in the transarterial embolization of fibroids.

\section{Materials and methods}

Study design. The present study was approved by the Institutional Review Board and Ethics Committee of the First People's Hospital of Changzhou (Changzhou, China). All patients provided written informed consent prior to participation. The study population was comprised of patients with symptomatic uterine fibroids who were scheduled to undergo transarterial UAE at the Department of Interventional Radiology at the First People's Hospital of Changzhou (Changzhou, China) between May 2016 and September 2018. Dyna CT and DSA were performed in all patients during angiographic embolization of the bilateral internal iliac arteries.

The inclusion criteria for the present study were as follows: i) Females aged $<50$ years of age with uterine fibroids (types I-III) as determined by the International Federation of Gynecology and Obstetrics assessed by B-ultrasound (17), sessile with intramural extension of fibroid $<50 \%$ (type I), sessile with intramural extension by $>50 \%$ (type II) or lesions localized in the myometrium with no deformation of the endometrial cavity (type III); ii) good general health; and iii) willing to undergo UAE. The exclusion criteria for the present study were as follows: i) Presence of other malignant or benign tumors; ii) tumors of $>10 \mathrm{~cm}$ in diameter or non-submucosal myomas $<3 \mathrm{~cm}$ in diameter; iii) presence of endometriosis or adenomyosis; iv) acute or chronic inflammation of the pelvic cavity; v) allergy to the contrast agents used during intervention therapy; and vi) patient age $>50$ years.

Angiography. Angiography was performed on the Siemens Artis zeego Syngo Dyna CT 360 digital angiography system (Siemens Medical Solutions) with a $\mathrm{C}$-arm rotational speed of 30 degrees/sec and a rotational range of 200 degrees.

The operation was performed by a single physician with 10 years of experience at the Department of Interventional Radiology of the First People's Hospital of Changzhou (Changzhou, China). The diameter of the image intensifier on the $\mathrm{C}$-arm was 16 inches. The Dyna CT software, non-ionic iodinated contrast agent (Omnipaque; GE Healthcare) and high-pressure syringe (Mark V Provis; Medrad, Inc.) were used as described below.

For angiography, the Seldinger technique was implemented using a $5 \mathrm{~F}$ sheath (18). The 5F-RH catheter was placed in the bilateral internal iliac arteries for Dyna CT imaging of the bilateral internal iliac arteries, after confirming the origin of the bilateral uterine arteries by Dyna CT 3-D angiography.
The contrast agent was injected into patients to observe the vascular architecture of the uterine arteries and fibroid staining. Using the contrast agent Omnipaque $(300 \mathrm{mg} / \mathrm{ml}$; internal iliac arteries, $6 \mathrm{ml}$ at $1 \mathrm{ml} / \mathrm{sec}$ ), the DSA scan was performed $2 \mathrm{sec}$ after starting the injection, with an acquisition frame rate of 4 frames/sec, collection matrix of 1,024 by 1,024 and acquisition time of 4 sec.

Masked images were obtained with a 360-degree rotation of the $\mathrm{C}$-arm, followed by a return to the start position. The patients included in the present study were $<50$ years of age and were able to effectively hold their breath, which minimized the risk of motion artifacts. The whole procedure lasted only $7 \mathrm{sec}$ and only a short breath hold was required, which minimized the number of artifacts.

Since the present study aimed to assess the usefulness of Dyna CT angiography in transarterial UAE of fibroids, it was important to visualize the uterus and fibroid. Therefore, it was necessary to image the uterine arteries and stained fibroids during rotational DSA. To fulfill this requirement, the timing of contrast agent injection and the amount of agent injected were determined based on previously performed internal iliac artery angiography, as an equal amount was required for the contrast media to fill the artery and reveal fibroid staining throughout the entire rotational DSA session. During internal iliac artery angiography, the time elapsed until visualization of the stained fibroids on the DSA images was determined and the start of contrast agent injection preceded the start of rotational DSA by this amount of time. The contrast agent injection continued until the end of the rotational DSA. The contrast agent for Dyna CT was Omnipaque (300 mg/ml; bilateral internal iliac, $3 \mathrm{ml} / \mathrm{sec}$; imaging was performed $2 \mathrm{sec}$ after the start of contrast agent injection). Imaging data from Dyna CT were automatically transferred to the Siemens X-Leonardo image processing workstation (Siemens Medical Solutions) for vascular imaging processing with volume-rendering technology. This allowed for better visualization of the origin of uterine arteries and fibroids with an acquisition frame rate of 60 frames/sec and a matrix size of 1,024 by 1,024 . Although the density resolution of Dynamic $\mathrm{CT}$ is lower than that of conventional $\mathrm{CT}$, the radiation dose was lower than that of conventional CT. Volume-rendering technology was used to locate the origin of uterine arteries and fibroids during transarterial UAE of the fibroids.

Contrast agent and radiation dose. The radiation dose of Dyna CT was $233 \mathrm{mGy}$ per scan with a bilateral dose of 466 mGy. The DSA contrast agent (Omnipaque) was given manually at a volume of $5-8 \mathrm{ml}$, with a bilateral volume of 10-16 $\mathrm{ml}$. Since the location and angle of the tube were adjusted for each patient during surgery, the operation times varied between patients. The radiation doses given to patients were between 200 and $500 \mathrm{mGy}$.

Image analysis. The visualization qualities of the origin of uterine arteries and stained fibroids imaged by Dyna CT 3-D rotational angiography and DSA anterior-posterior images were compared. DSA anterior-posterior images were evaluated at the workstation, while Dyna CT images were assessed by rotating the images created using volume-rendering technology. 3-D rotational angiography images were available for 
examination at the workstation immediately after completing the Dyna CT scans. The preparation of volume-rendering technology images required $1 \mathrm{~min}$. The volume-rendering technology images are the most straightforward reconstructed images. Two experts in angiography ( $\mathrm{ZHH}$ and QW) assessed the origin of uterine arteries and stained fibroids independently and any discrepancies were resolved by consensus.

Statistical analysis. Continuous data were expressed as the mean \pm standard deviation and enumeration data as $n(\%)$. Data were compared by the McNemar test using SPSS software (v19.0, IBM Corp.). $\mathrm{P}<0.05$ was considered to indicate statistical significance.

\section{Results}

Baseline characteristics of the patients. A total of 74 patients were considered for the present study and 9 were excluded based on the inclusion and exclusion criteria. Finally, 65 patients were included in the study. The average age of the patients was $38.5 \pm 9.5$ years. A total of 21 patients had type I submucosal myomas, 14 had type II submucosal myomas, 19 had intramural myoma (type III) and 11 patients had mixed myomas (types I-III). The detailed patient characteristics are summarized in Table I.

Image processing in different modalities. Dyna CT imaging was successfully completed in all of the patients. The 3-D rotational angiographic images, including the volume-rendering technology images, are superior for 3-D visualization of specific vascular structures of the uterine arteries (19), particularly for identifying 3-D structures of the origin of uterine arteries in patients where DSA anterior-posterior images display complex pelvic vessel anatomy or overlapping arteries (Figs. 1 and 2). Maximum intensity projection images and shaded surface display images may display the 3-D structures of arteries, yet volume-rendering technology images are more efficient for identifying the 3-D structures of arterial branches (15).

Outcomes of Dyna CT angiography vs. DSA-AP imaging. Dyna CT 3-D angiography displayed the origin of uterine arteries in all patients (100\%), while DSA anterior-posterior only displayed the origin in 45 cases $(69.2 \%, \mathrm{P}=0.03)$. There were 12 cases with overlapping uterine arteries and 8 cases with complex vascular anatomies. However, the detection rate of fibroids on Dyna CT 3-D angiography was lower than that on DSA anterior-posterior imaging (Fig. 3). Stained fibroids were identified in 57 cases $(87.7 \%)$ by Dyna CT and in all 65 cases $(100 \%, \mathrm{P}=0.03)$ by DSA anterior-posterior imaging. There were statistically significant differences in the detection rates for fibroids and the origin of bilateral uterine arteries between the two imaging modalities (Table II).

\section{Discussion}

In the present study, Dyna CT was superior to DSA anterior-posterior imaging in detecting the origin of bilateral uterine arteries, particularly in cases with overlapping uterine arteries on DSA anterior-posterior images or in patients with complex vascular anatomies. However, Dyna CT was less
Table I. Patient baseline characteristics.

\begin{tabular}{lc}
\hline Variable & Value \\
\hline Age (years) & $38.5 \pm 9.5$ \\
Type of fibroid & \\
I & $21(32.3)$ \\
II & $14(21.5)$ \\
III & $19(29.3)$ \\
Mixed & $11(16.9)$ \\
Previous history of uterine surgery & \\
Yes & $23(35.4)$ \\
No & $42(64.6)$ \\
Dysmenorrhea & \\
Yes & $25(38.5)$ \\
No & $40(61.5)$ \\
Development of the ovarian branch & \\
of the uterine artery & \\
Bilateral & $12(18.5)$ \\
Unilateral & $11(16.9)$ \\
None & $42(64.6)$ \\
\hline
\end{tabular}

Values are expressed as $\mathrm{n}(\%)$ or the mean \pm standard deviation.

efficient in detecting stained fibroids. To the best of our knowledge, there are no other studies on whether Dyna CT was superior to DSA imaging in detecting the origin of bilateral uterine arteries.

Due to recent technological advances in angiography, it is now possible to perform rotational DSA using a C-arm equipped with a large diameter image intensifier, which may be used to image pelvic vascular structures (19). A fundamental requirement of imaging procedures for transarterial UAE of fibroids is angiography, yet the understanding of the spatial distribution of vascular structures is highly dependent on the physician's anatomical knowledge, since the images are 2-D (7). Any method that relies heavily on the expertise and experience of physicians may have limited utility in the clinic, as misunderstandings are more likely to occur (15). Transarterial UAE of fibroids requires a detailed understanding of 3-D vascular anatomy prior to embolization. For this reason, 3-D angiography using rotational DSA during pelvic angiography was performed for transarterial embolization of fibroids and its usefulness in transarterial embolization of fibroids was assessed.

The success of transarterial UAE of fibroids depends on precisely identifying the origin of uterine arteries, along with highly selective catheterization (20). Previous studies have indicated that excessive embolization may damage normal myometrium, ovaries or fallopian tubes, resulting in uterine necrosis, infections or ovarian failure in rare cases. However, incomplete therapy or further blood supply to the tumor may result in treatment failure (21). Angiography images are generally 2-D, yet it is essential to assess the 3-D anatomy of the vasculature in patients with overlapping vessels or complex vascular structures $(19,22)$. In the present study, Dyna CT 


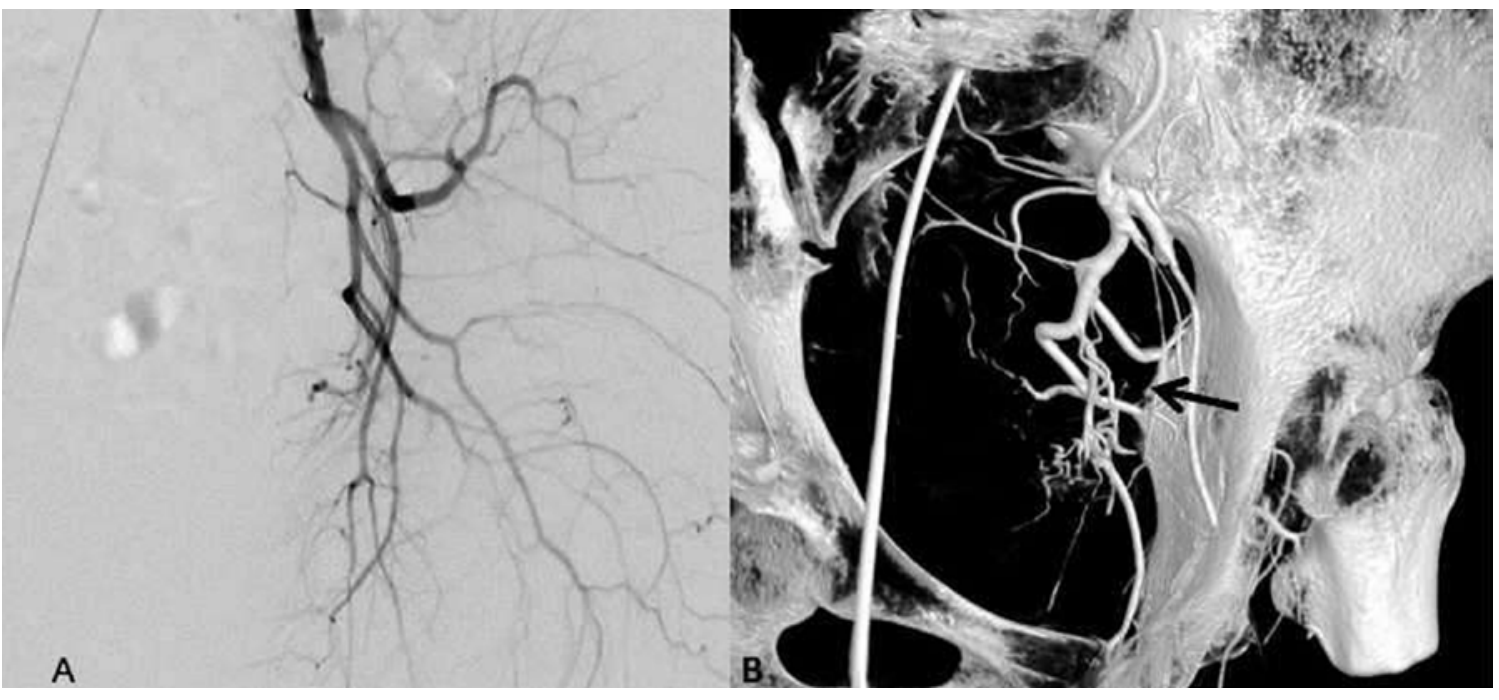

Figure 1. Representative case of a 35-year-old female with symptomatic submucosal fibroids. (A) Angiography of the right internal iliac arteries (DSA-AP image). Since the internal iliac artery branches overlapped, it was not possible to clearly identify the uterine artery on DSA-AP images. (B) Volume rendering reconstruction of Dyna CT 3-dimensional angiography, right anterior oblique 45-degree image. The overlapping uterine arteries (black arrows) in the AP view were clearly identified. DSA-AP, digital subtraction angiography anterior-posterior.

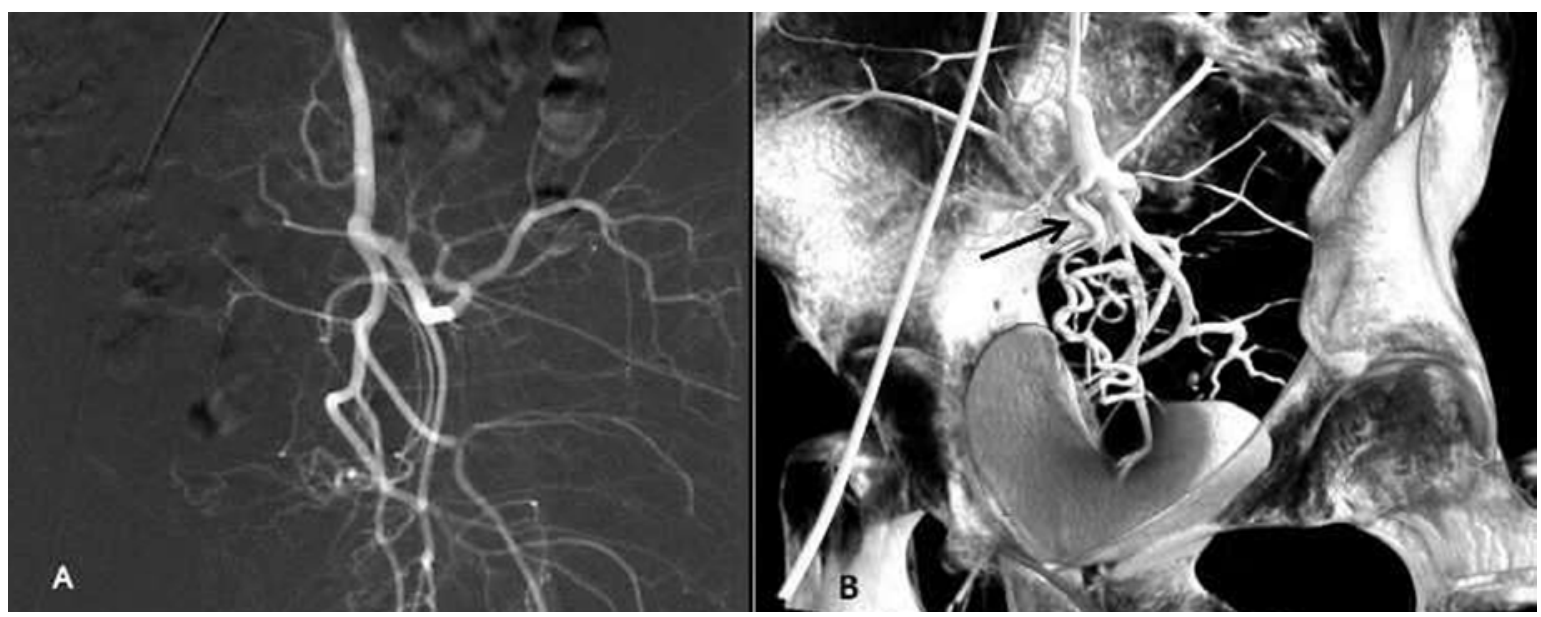

Figure 2. Representative case of a 32-year-old female with symptomatic mixed fibroids. (A) Angiography of the left internal iliac arteries (DSA-AP image). Since the internal iliac artery branches overlapped, the uterine artery was not observed on the DSA-AP images. (B) Volume rendering reconstruction of Dyna CT 3-dimensional angiography, right anterior oblique 45-degree image. After contrast agent injection into the left uterine artery, the branching features of the left internal iliac artery were clearly visualized in the anterior region and the overlapped uterine arteries (black arrows) in the AP view were clearly identified. DSA-AP, digital subtraction angiography anterior-posterior.

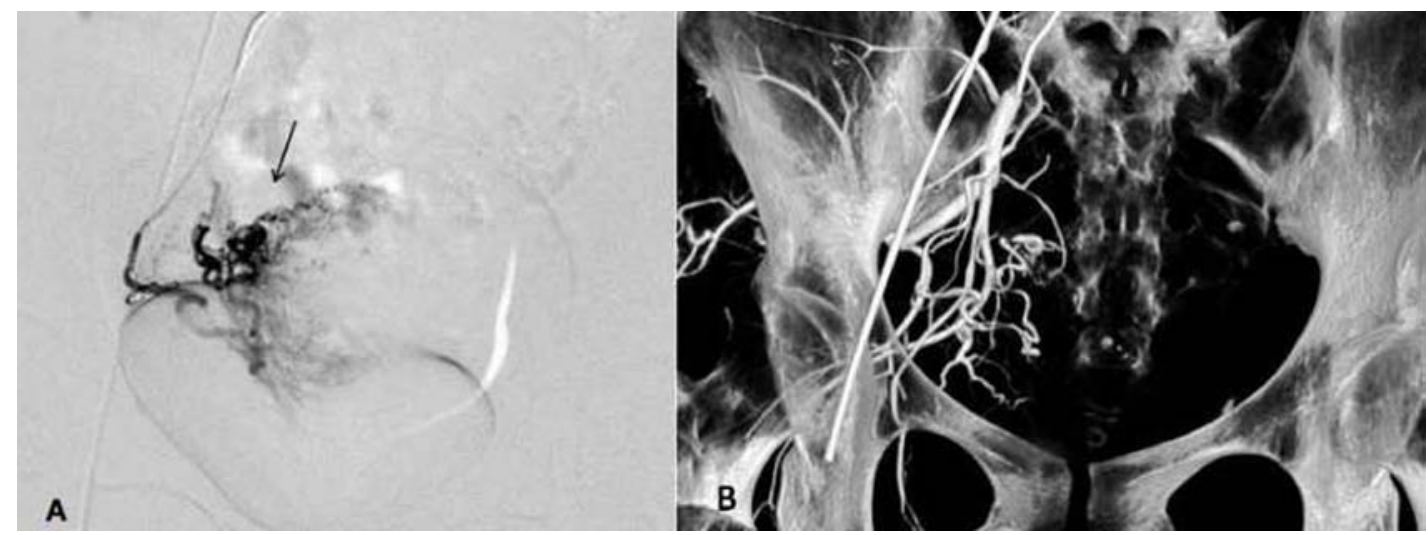

Figure 3. Representative case of a 32-year-old female with symptomatic mixed fibroids. (A) Angiography of the right internal iliac arteries (DSA-AP image). Uterine staining was observed, as well as the outline of the uterus. (B) Volume rendering reconstruction of Dyna CT 3-D angiography after contrast agent injection in the right internal iliac artery. The origin of the uterine artery was clearly visualized. However, uterine staining observed on DSA-AP imaging (A, arrows) was not detected by Dyna CT 3-D angiography. DSA-AP, digital subtraction angiography anterior-posterior; 3-D, 3-dimensional. 
Table II. Outcomes of Dyna CT angiography vs. DSA-AP imaging.

\begin{tabular}{lccc}
\hline Detected item & Dyna CT & DSA-AP & P-value \\
\hline $\begin{array}{l}\text { Origin of bilateral uterine } \\
\text { arteries (n=65) }\end{array}$ & $65(100)$ & $45(69.2)$ & 0.03 \\
Fibroids (n=65) & $57(87.7)$ & $65(100)$ & 0.03 \\
\hline
\end{tabular}

Values are expressed as $\mathrm{n}(\%)$. AP, anterior-posterior; DSA, digital subtraction angiography.

detected the origin of bilateral arteries in all of the patients, while DSA anterior-posterior images were unable to detect the origin in all of the patients, corroborating the notion that Dyna CT may be useful in patients with overlapping uterine arteries or complex vascular anatomies on DSA anterior-posterior images.

In a previous study, Gupta et al (23) demonstrated that 3-D rotational angiography is preferable to 2-D techniques for UAE. When using 3-D angiography by Dyna CT in the transarterial embolization of fibroids, it was esteemed to overcome the limits of DSA anterior-posterior imaging. As the ultimate objective, it was evaluated whether 3-D rotational angiography may be used in place of DSA anterior-posterior imaging by assessing the status of stained fibroids. Compared with DSA anterior-posterior imaging, Dyna CT demonstrated inferior visualization quality for the stained fibroids. In certain patients, fibroids were visualized by DSA anterior-posterior imaging, but not by Dyna CT 3-D rotational angiography.

One reason for this finding may be the difficulty in simultaneously visualizing the origin of uterine arteries and fibroids. During Dyna CT, the internal iliac artery and fibroids are filled with contrast media. Therefore, the time from the start of contrast agent injection to the visualization of stained fibroids in DSA imaging during internal iliac artery angiography is measured and Dyna CT is initiated when the fibroid staining begins to appear. In return, contrast agent injection is commenced prior to Dyna CT and it is continuously administered until completion. However, this increases the concentration of contrast agent in the uterus and may lead to poor fibroid visualization. In addition, there was a difference in radiation dose required for each frame. The radiation dose is measured as the mass voltage $(\mathrm{V})$ and the mass is measured by current (mA) multiplied by time (sec). In Dyna CT, the X-ray tube rotates to restrict the exposure time. For compensation, the current $(\mathrm{mA})$ increases within a specific limit, which increases the voltage and light scatter and decreases the image contrast. Motion artifacts may be another factor. 3-D angiography by Dyna CT does not require the patients to hold their breath, since it is performed in the head region. However, in the abdominal region, the patient must hold his/her breath for at least $7 \mathrm{sec}$ from the start of masked images to the end of rotational DSA and another $2 \mathrm{sec}$ for contrast agent injection $(24,25)$. In the present study, poor image quality of the fibroids was noted directly below the bottom of the uterus. This may be a result of motion artifacts due to patients not adequately holding their breath.

In the present study, patients over the age of 50 years were excluded, as older females experience menopause, which frequently results in the shrinkage of fibroids, reducing clinical symptoms. US is the gold standard as it allows physicians to accurately visualize the location and size of fibroids. Pathological diagnoses require highly invasive biopsies or surgeries, which should be avoided if possible. The present study had certain limitations that should be addressed. First, selection bias may have been present due to patients being selected from a single center. In addition, the sample size was relatively small. Further studies with larger sample sizes using patients from multiple clinical centers are required to confirm the present results.

In conclusion, 3-D angiography by Dyna CT is useful for identifying the 3-D anatomy of the bilateral internal iliac arteries during the transarterial UAE of fibroids, particularly in patients with the origin of uterine overlapping on DSA anterior-posterior images and patients with complex vascular branches. Compared with DSA anterior-posterior imaging, Dyna CT exhibited reduced visualization quality for fibroids. Therefore, Dyna CT cannot replace DSA anterior-posterior imaging for procedural evaluation of patients during transarterial embolization of fibroids. However, Dyna CT may provide information supplementary to DSA anterior-posterior images, which may assist physicians during treatment planning.

\section{Acknowledgements}

The authors wish to thank the patient advisers (Mrs. Chun-lan $\mathrm{Xu}$, Mrs. Hui Wang and Dr Yuanquan Huang from the First People's Hospital of Changzhou, Changzhou, Jiangsu, China) for their assistance.

\section{Funding}

The present study was supported by the Natural Science Foundation of Jiangsu Province (grant no. BK20180185) and the Youth Foundation of Changzhou First People's Hospital (grant no. yy2017006).

\section{Availability of data and materials}

The datasets generated and analyzed during the present study are available from the corresponding author on reasonable request.

\section{Authors' contributions}

CYW, JGX and WHC performed most of the investigations, the data analysis and wrote the manuscript; JGX supervised CYW and WHC during the UAE procedure. YFL provided assistance during the UAE procedure and performed statistical analysis. CYW, ZHH and QW contributed to the interpretation of the data and analyses. All of the authors read and approved the manuscript.

\section{Ethics approval and consent to participate}

The present study was approved by the Institutional Review Board and Ethics Committee of the First People's Hospital of 
Changzhou (Changzhou, China). All procedures performed in studies involving human participants were in accordance with the ethical standards of the institutional and/or national research committee and with the 1964 Helsinki declaration and its later amendments or comparable ethical standards. All patients provided written informed consent prior to participation.

\section{Patient consent for publication}

All data of the present study were published with the written informed consent of the patients.

\section{Competing interests}

The authors declare that they have no competing interests.

\section{References}

1. Stewart EA: Clinical practice. Uterine fibroids. N Engl J Med 372: 1646-1655, 2015.

2. Olejek A, Olszak-Wasik K and Czerwinska-Bednarska A: Long-term intermittent pharmacological therapy of uterine fibroids-a possibility to avoid hysterectomy and its negative consequences. Prz Menopauzalny 15: 48-51, 2016.

3. Zhao H, Li Y, Xu Q, Peng F, Zhao J, Webb RC, Peng C and Yu C: Establishment of a rat model for uterine leiomyomas based on western and traditional Chinese medicine theories. Braz J Med Biol Res 51: e7627, 2018.

4. Bulun SE: Uterine fibroids. N Engl J Med 369: 1344-1355, 2013.

5. Mas A, Tarazona M, Dasí Carrasco J, Estaca G, Cristóbal I and Monleón J: Updated approaches for management of uterine fibroids. Int J Womens Health 9: 607-617, 2017.

6. Ananthakrishnan G, Murray L, Ritchie M, Murray G, Bryden F, Lassman S, Lumsden MA and Moss JG: Randomized comparison of uterine artery embolization (UAE) with surgical treatment in patients with symptomatic uterine fibroids (REST trial): Subanalysis of 5-year MRI findings. Cardiovasc Intervent Radiol 36: 676-681, 2013.

7. Gupta JK, Sinha A, Lumsden MA and Hickey M: Uterine artery embolization for symptomatic uterine fibroids. Cochrane Database Syst Rev CD005073: 2014.

8. Sapoval M, Pellerin O, Rehel JL, Houdoux N, Rahmoune G, Aubert B and Fitton I: Uterine artery embolization for leiomyomata: Optimization of the radiation dose to the patient using a flat-panel detector angiographic suite. Cardiovasc Intervent Radiol 33: 949-954, 2010

9. Ko HK, Shin JH, Ko GY, Gwon DI, Kim JH, Han K and Lee SW: Efficacy of prophylactic uterine artery embolization before obstetrical procedures with high risk for massive bleeding. Korean J Radiol 18: 355-360, 2017.

10. Mara $M$ and Kubinova K: Embolization of uterine fibroids from the point of view of the gynecologist: Pros and cons. Int $\mathrm{J}$ Womens Health 6: 623-629, 2014.

11. Salazar GM, Gregory Walker T, Conway RF, Yeddula K, Wicky S, Waltman AC and Kalva SP: Embolization of angiographically visible type I and II utero-ovarian anastomoses during uterine artery embolization for fibroid tumors: Impact on symptom recurrence and permanent amenorrhea. J Vasc Interv Radiol 24: 1347-1352, 2013.
12. Firouznia K, Ghanaati H, Sharafi A, Abahashemi F, Hashemi H, Jalali AH and Shakiba M: Comparing ovarian radiation doses in flat-panel and conventional angiography during uterine artery embolization: A randomized clinical trial. Iran J Radiol 10: 111-115, 2013.

13. Maleux G, Michielsen K, Timmerman D, Poppe W, Heye S, Vaninbroukx $\mathbf{J}$ and Bosmans $\mathrm{H}$ : 2D versus 3D roadmap for uterine artery catheterization: Impact on several angiographic parameters. Acta Radiol 55: 62-70, 2014.

14. Fahrig R, Dixon R, Payne T, Morin RL, Ganguly A and Strobel N: Dose and image quality for a cone-beam C-arm CT system. Med Phys 33: 4541-4550, 2006.

15. Wang C, Wang Q, Chen W, He Z, Huang Y, Lu Y and Miao Y: Dyna CT arteriographic evaluation of hepatocellular carcinoma for treatment by trans-catheter arterial chemoembolization. Int J Clin Exp Med 8: 20548-20555, 2015.

16. Colombo F, Cavedon C, Francescon P, Casentini L, Fornezza U, Castellan L, Causin F and Perini S: Three-dimensional angiography for radiosurgical treatment planning for arteriovenous malformations. J Neurosurg 98: 536-543, 2003.

17. Doherty L, Mutlu L, Sinclair D and Taylor H: Uterine fibroids: Clinical manifestations and contemporary management. Reprod Sci 21: 1067-1092, 2014

18. Guan YS, He Q and Wang MQ: Transcatheter arterial chemoembolization: History for more than 30 years. ISRN Gastroenterol 2012: 480650, 2012.

19. Tanigawa N, Komemushi A, Kojima H, Kariya S and Sawada S: Three-dimensional angiography using rotational digital subtraction angiography: Usefulness in transarterial embolization of hepatic tumors. Acta Radiol 45: 602-607, 2004.

20. Czuczwar P, Woźniak S, Szkodziak P, Woźniakowska E, Paszkowski M, Wrona W, Milart P, Paszkowski T and Popajewski M: Predicting the results of uterine artery embolization: Correlation between initial intramural fibroid volume and percentage volume decrease. Prz Menopauzalny 13: 247-252, 2014.

21. Pelage JP, Cazejust J, Pluot E, Le Dref O, Laurent A, Spies JB, Chagnon $S$ and Lacombe P: Uterine fibroid vascularization and clinical relevance to uterine fibroid embolization. Radiographics 25 (Suppl 1): S99-S117, 2005.

22. Lanzavecchia S, Bellon PL and Radermacher M: Fast and accurate three-dimensional reconstruction from projections with random orientations via radon transforms. J Struct Biol 128: 152-164, 1999.

23. Gupta A, Zuurmond K, Grünhagen T and Maleux G: Threedimensional rotational angiography is preferable to conventional two-dimensional techniques for uterine artery embolization. Diagn Interv Radiol 19: 418-422, 2013.

24. Tacher V, Radaelli A, Lin M and Geschwind JF: How I do it: Cone-beam CT during transarterial chemoembolization for liver cancer. Radiology 274: 320-334, 2015.

25. Huber ME, Hengesbach D, Botnar RM, Kissinger KV, Boesiger P, Manning WJ and Stuber M: Motion artifact reduction and vessel enhancement for free-breathing navigator-gated coronary MRA using 3D k-space reordering. Magn Reson Med 45: 645-652, 2001.

This work is licensed under a Creative Commons Attribution-NonCommercial-NoDerivatives 4.0 International (CC BY-NC-ND 4.0) License. 